\title{
2D Reflectometry for the investigation of polymer interfaces: off-specular neutron scattering
}

\author{
Aljoša Hafner ${ }^{a}, \varsubsetneqq$, Philipp Gutfreund ${ }^{a}$, Boris P. Toperverg ${ }^{a, c}$, \\ Mark Geoghegan ${ }^{d} \S$, and Michele Sferrazza ${ }^{b}$ \\ ${ }^{a}$ Institut Laue - Langevin, 71 avenue des Martyrs, CS 20156, 38042 Grenoble, Cedex \\ 9, France \\ ${ }^{b}$ Université Libre de Bruxelles, Département de Physique, 1050 Brussels, Belgium \\ ${ }^{c}$ Petersburg Nuclear Physics Institute, National Research Center "Kurchatov \\ Institute",188300 Gatchina, Russia \\ ${ }^{d}$ Department of Physics and Astronomy, University of Sheffield, Hicks Building, \\ Hounsfield Road, Sheffield S3 7RH, United Kingdom \\ E-mail: gutfreund@ill.fr
}

\begin{abstract}
Specular and off-specular neutron reflectometry have been used in a combined approach to study thin polymer films. Our goal in this work is to illustrate the power of the off-specular scattering technique to probe the properties of the buried interface of immiscible polymer bilayers of deuterated polystyrene (dPS) and protonated poly(methyl methacrylate) (h-PMMA). The diffuse scattering stemming from these systems is discussed in relation to thermal fluctuations at the polymer/polymer interface, providing a means to extract in-plane correlation lengths from buried interfaces. In addition the onset of hole formation in the top layer is evidenced by the diffuse scattering, not easily detectable by specular reflection alone.
\end{abstract}

Keywords: Neutron reflectometry, Off-specular scattering, Thin polymer films, DWBA

\section{Introduction}

One of the challenges related to the study of buried interfaces is the relative inaccessibility of their properties. Due to their non-destructive nature and the ability to selectively deuterate different molecular species, neutrons are suitable for probing buried polymer-polymer and/or polymer-solid interfaces. With the use of specular and off-specular neutron reflectivity (SNR and OSS), we are able to access two length scales at the same time: The density profile perpendicular to the sample surface, yielding the thickness of the films and the roughness at their interfaces with $\sim \AA$ accuracy (specular $\mathrm{NR}$ ), and the structure parallel to the sample surface, yielding in-plane correlation

$\ddagger$ Current address: CERIC-ERIC, 34149 Basovizza, Trieste, Italy

$\S$ Current address: School of Engineering, Newcastle University, Merz Court, Newcastle upon Tyne, NE1 7RU, UK 
lengths of density variations in the range from $\sim 10 \mathrm{~nm}$ to $\sim 10 \mu \mathrm{m}$ (OSS) [1]. However, one critical point is the limit of detection of variations of volume density: in fact if these changes are only marginal relative to the rest of the layer, they would, most probably, be within the experimental error if using only specular reflectivity. We argue here that for a full understanding of the properties of the interface we need to link specular and off-specular scattering components.

SNR is a well established technique and nowadays there is a multitude of readily available software and established procedures for detailed analysis of the reflectivity.|I It gives information on the density profile perpendicular to the sample plane. Structures parallel to the sample surface are more difficult to access since the information resides in the scattering in the off-specular direction and this is much less intense. For this reason OSS studies on thin films of soft matter have been mostly performed by synchrotron X-ray reflectometry (XRR) providing much higher flux [2, 3, 4, 5].

The other, more important, reason for the limited use of OSS in neutron scattering is that there is not as much standard software available for the analysis of OSS. This is partly due to the complexity of description of the processes happening in the close vicinity to the critical angle going far beyond the usually used Born approximation [1]. Most of the present understanding and software tools for grazing incidence techniques stem from research done with X-rays. Despite being very similar to neutrons, there are some important differences, which have to be taken into account. Contrary to general belief, the OSS carries not only the lateral structural information, but is also heavily dependent on the neutron wave field distribution inside the film. It thus constrains the fitting of SNR, similar to an additional scattering contrast. It is therefore important to devise a description which links SNR and OSS without any scaling parameters and with minimal extra parameters characterizing OSS.

In this work, we illustrate how the OSS technique combined with SNR allows an understanding of the properties of soft matter thin films. To this goal we have considered an immiscible polymer/polymer interface as a model system by preparing bilayers of deuterated polystyrene (d-PS) and protonated poly(methyl methacrylate) (h-PMMA) of different thickness supported by Si substrates. For this system in equilibrium at a temperature $T$ we can predict the properties of the interface between the two polymers: the interface width $\sigma$ corresponds to the squared sum [6] of the intrinsic roughness $\sigma_{\mathrm{i}}$, coming from the Flory-Huggins $(\mathrm{FH})$ interaction parameter $\chi$ and scaling with the monomer size $a$ [7], and of the capillary wave (CW) roughness related to the interfacial tension $\gamma$ [8]:

$$
\sigma^{2}=\sigma_{\mathrm{i}}^{2}+\frac{k_{\mathrm{B}} T}{2 \pi \gamma} \ln \left(\frac{q_{\max }}{q_{\min }}\right),
$$

where the capillary wave spectrum is cut at low and high wave numbers $q_{\min }$ and $q_{\max }$, respectively. $k_{\mathrm{B}}$ denotes the Boltzmann constant.

The intrinsic width, which is on the order of $\sigma_{\mathrm{i}}=2 \mathrm{~nm}$ [9, 10] is responsible for the

\|l http://www.reflectometry.net/reflect.htm\#Analysis 
short wavelength cut-off of the $\mathrm{CW}$ spectrum and is related to this intrinsic interface width by: $q_{\max }=2 \pi / \sigma_{\mathrm{i}}$. Note that we use the Gaussian sigma width in this work, often the tanh width is used to describe the polymer/polymer interface extend [11], however, those two are just related by a constant factor of $\sqrt{2 / \pi}$.

The long wavelength cut-off can be of different origin: For bulk liquids very large wavelengths will be suppressed by gravity and cause a cut-off $a_{\text {grav }}=2 \gamma / g \Delta \rho$, with $g$ being the gravitational acceleration and $\Delta \rho$ the difference in mass densities of two phases. This wavelength is on the mm length scale for organic materials. For thin films of thickness $d$ much smaller than this capillary length, van der Waals interactions with the substrate may become significant and the low wavevector cut-off then comes from the dispersive capillary wave term [9]. This length is usually on the micrometre or submicrometre scale and was used to estimate $q_{\min }$ in this study. For polymer thin films, non-liquid like behaviour is found with a smaller cut-off than the vdW cut-off. This is assumed to be due to chain adsorption to a non-repulsive substrate suppressing large amplitudes even further [3, 5].

In the case of scattering experiments, only surface undulations with wavelengths shorter than the coherence length of the radiation can be observed. Therefore the high wavelength cut-off may not be detectable and the spectrum is cut at the coherence length, which can vary between dozens of microns (neutron reflectometry) and many hundred microns (synchrotron XRR), depending on the instrument used.

The resulting in-plane structure of CWs is modeled by a Lorentz function in reciprocal space as done for OSS studies with neutrons on lipid bilayers [12] before. We extend this to buried polymer/polymer interfaces in this work and compare the results to X-ray studies looking at the surface of thin polymer layers [3, 4, 5]. By carefully combining the analysis of the reflectivity and the out-of-reflection scattering, we can be able to detect more precise information on the structure of the buried interface.

\section{Experimental details}

\subsection{Sample preparation}

The two polymers used are deuterated polystyrene (d-PS) and poly(methyl methacrylate) (h-PMMA). Both of the polymers were obtained from Polymer Source, Inc. and had a weight-averaged molecular weight of $M_{w}=252 \mathrm{kDa}$ for d-PS and $M_{w}=342.9 \mathrm{kDa}$ for h-PMMA, respectively. All the polymers used had a dispersity of at most 1.11 .

The silicon substrates were $5 \mathrm{~cm}$ in diameter and $0.5 \mathrm{~cm}$ thickness. One side was polished and had a native oxide layer $\left(\mathrm{SiO}_{2}\right)$ of thickness of around $2 \mathrm{~nm}$ and a roughness of around $0.5 \mathrm{~nm}$. The thickness of the $\mathrm{SiO}_{2}$ was measured with ellipsometry (Beaglehole Picometer) prior to polymer deposition. The surfaces were cleaned by ultrasonication in several organic solvents and Milli-q water, leading to a surface energy of $26 \mathrm{~mJ} / \mathrm{m}^{2}$ [13]. 
Table 1: List of samples with their structural properties extracted from specular NR. All samples were annealed at $160^{\circ} \mathrm{C}$ for $48 \mathrm{~h}$ for the PS layer $+12 \mathrm{~h}$ in the final bi-layer configuration. $d_{\mathrm{dPS}}, d_{\mathrm{hPMMA}}, N b_{\mathrm{dPS}}$ and $N b_{\mathrm{hPMMA}}$ represent the thicknesses and SLDs of the respective layers. $\zeta$ and $\eta$ are the width and asymmetry parameter, respectively, of the asymmetric tanh function describing the interface as defined by equation 2.61 in Ref. [15] (p. 22-24, figure 2.13, Eqs. 2.52-2.61) and described in sec.3.1] in this work. Uncertainties are noted in parentheses.

\begin{tabular}{ccccccc} 
Name & $d_{\mathrm{dPS}}[\AA]$ & $d_{\mathrm{hPMMA}}[\AA]$ & $N b_{\mathrm{dPS}}\left[10^{-6} \AA^{-2}\right]$ & $N b_{\mathrm{hPMMA}}\left[10^{-6} \AA^{-2}\right]$ & $\zeta[\AA]$ & $\eta$ \\
\hline $\mathrm{A}$ & $892.0(5)$ & $1221(13)$ & $6.33(2)$ & $1.059(3)$ & $23.0(1)$ & $-0.5(1)$ \\
$\mathrm{C}$ & $640.0(3)$ & $1062(9)$ & $6.32(3)$ & $1.08(4)$ & $22.7(2)$ & $-0.8(1)$ \\
$\mathrm{D}$ & $516.2(5)$ & $1107(13)$ & $6.34(6)$ & $1.048(6)$ & $21.2(3)$ & $-0.9(1)$ \\
$\mathrm{E}$ & $237.0(4)$ & $1191(6)$ & $6.33(1)$ & $1.08(7)$ & $20.3(2)$ & $-0.9(1)$ \\
$\mathrm{F}$ & $130.9(6)$ & $1101(5)$ & $6.41(4)$ & $1.10(8)$ & - & - \\
\hline
\end{tabular}

To prepare the polymer bilayer the bottom d-PS layer was first spin coated onto the silicon substrates from toluene solutions of different concentrations in order to obtain different thicknesses from 120-1200 $\mathrm{A}$. The bottom d-PS layer was then annealed in a vacuum oven for $48 \mathrm{~h}$ at $T=160^{\circ} \mathrm{C}$, which is well above the glass transition temperature of both polymers, $T_{g}=95^{\circ} \mathrm{C}$ for PS [14] and $T_{g}=105^{\circ} \mathrm{C}$ for PMMA [14]. After the preannealing step, the h-PMMA layer of $\sim 1100 \AA$ thickness was deposited on top. Here, the polymer film was spin-coated onto a glass slide, which was subsequently immersed in milliQ water at a shallow angle to allow the PMMA layer to detach and float on the surface. It can then be picked up from below with the substrate with previously spin coated d-PS to create a bilayer. The resulting bilayer was subsequently annealed at $T=160{ }^{\circ} \mathrm{C}$ for 12 more hours. All samples prepared for this study are listed in table 1 .

Spin-coating was done on a Delta6 Süss MicroTec spinning at $500 \mathrm{rpm}$ for $2 \mathrm{~s}$, followed by $3000 \mathrm{rpm}$ for $55 \mathrm{~s}$. The target layer thickness was varied by varying the polymer concentration of the deposited solution, which was filtered with a polytetrafluoroethylene (PTFE) filter of pore size of around $0.5 \mu \mathrm{m}$. For both polymers, polystyrene (PS) and poly(methyl methacrylate) (PMMA), toluene was used as a solvent. All the solutions were prepared at least $24 \mathrm{~h}$ in advance to allow for proper dissolution. After each spin coating, as well as the final bilayer, the layers were individually investigated for imperfections by eye and by variable angle ellipsometry, in order to achieve the correct concentration of the toluene solution for the experiments and to verify whether all the layers were properly deposited.

\subsection{Neutron Scattering}

The kinetics of interface formation were monitored as a function of the annealing time with both specular neutron reflectometry and off-specular scattering that were performed on the D17 reflectometer in time-of-flight (ToF) mode [16] at the Institut Laue- 
Langevin. For NR the relative wavelength resolution $\Delta \lambda / \lambda$ and angular divergence $\Delta \theta_{\mathrm{i}} / \theta_{\mathrm{i}}$ were both fixed at $1 \%$, whereas for OSS $\Delta \lambda / \lambda$ was varied between $1 \%$ and $4 \%$ in the investigated q-range and $\Delta \theta_{\mathrm{i}} / \theta_{\mathrm{i}}=1.5 \%$. All resolutions are given in full width at half maximum (FWHM). In both cases the footprint on the sample was fixed to a square of $30 \mathrm{~mm} \times 30 \mathrm{~mm}$. The detector was $3.1 \mathrm{~m}$ away from the sample and had a pixel size of $1.2 \mathrm{~mm}$. SNR data was normalized to the incident beam spectrum by using COSMOS [17] and OSS data was normalized by using LAMP [18] and transformed into momentum space by using Överlåteren [19], if needed for visualization. OSS fitting was done in $\lambda$ vs. $2 \theta$ space using the here described algorithm[20]. SNR fitting was done in q-space with the algorithm described herein. Both SNR and OSS fitting was done after convoluting the theoretical scattering points by a Gaussian resolution function, whose width in q was provided by COSMOS for SNR and for the wavelength resolution in OSS fitting. The effective angular divergence in OSS fits, comprising the detector as well as incoming beam divergence, was extracted from a Gaussian fit to the specular peak on the $2 \mathrm{D}$ detector for wavelengths where the OSS signal was negligible.

\section{Experimental results}

\subsection{Specular Reflectivity model and results}

Samples with different thickness of the d-PS layer were measured (see figure 1a) in order to obtain the interfacial width as a function of layer thickness, i.e. the distance from the substrate.

In reflectometry measurements, only the squared modulus of the reflection amplitude is recorded, with phase information being lost. In order to establish the structure of a sample in real space, a model has to be constructed and then iteratively compared (fitted) to the data. For the samples presented here, there is an obvious choice of the model with the film composed of individual physical layers. The scattering contrast smearing over the interface region is modelled with the error function corresponding to a Gaussian distribution of height deviations from the ideal sharp interface. Additionally to the polymer layers, there is always a thin oxide layer covering bare Si substrates, which is typically around $\sim 20 \AA$ thick.

Therefore, a 3-layer model $\mathrm{SiO}_{2}$ / d-PS / h-PMMA was constructed in the beginning, with $\mathrm{Si}$ as a substrate and air as the top semi-infinite medium. While a 3-layer model including roughness at the interfaces is sufficient to describe the Kiessig fringes coming from the thicknesses of the top and bottom layers, it is unable to reproduce the fine modulation of some of the data, especially around the minima (see figure 2a). It turns out that in our case, a 3-layer model is insufficient in correctly describing this fine structure for some of the samples. As will be shown later, an additional interfacial layer, necessary to get a satisfactory specular fit, is also extremely important to account for the off-specular scattering intensity in the concerned samples. 


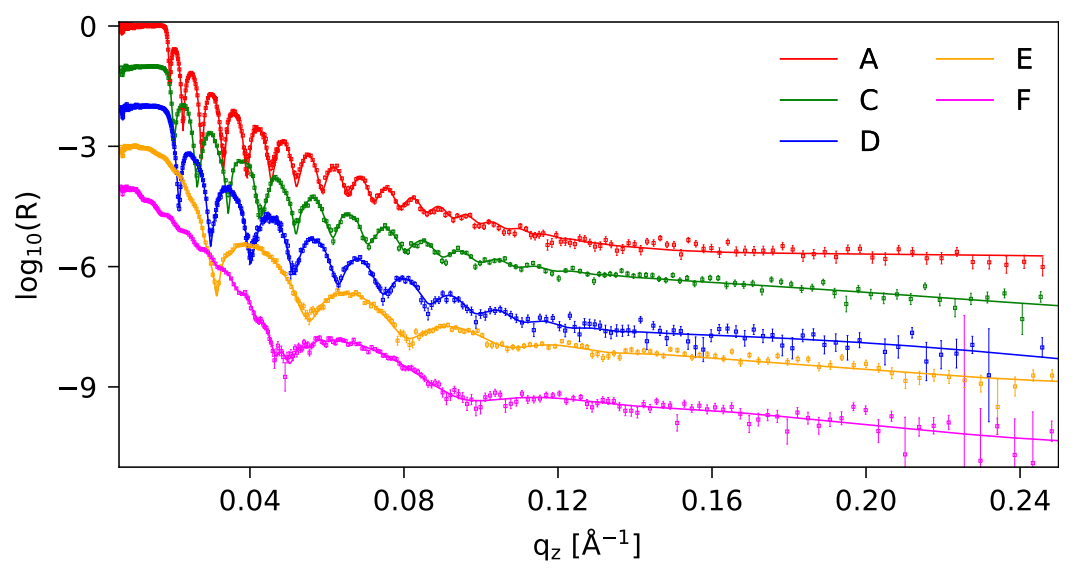

(a)

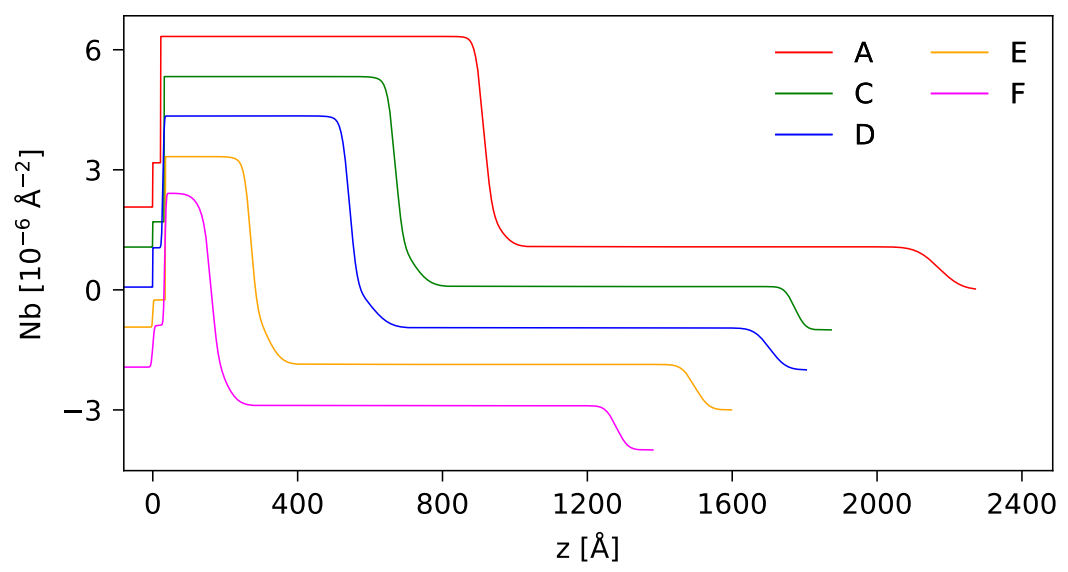

(b)

Figure 1: a) Logarithm of the specular reflectivity for the measured samples (points) with corresponding fits (lines). Curves are offset by -1 for clarity. b) Scattering length density profiles from the specular reflectivity fits in the same colour code. Curves are offset by $-10^{-6} \AA^{-2}$ for clarity. $z=0$ denotes the location of the $\mathrm{Si} / \mathrm{SiO}_{2}$ interface. All the sample parameters are listed in table 1 .

By introducing this additional layer, the tail region of the interface is effectively extended, showing an asymmetric shape. This is shown in figure $2 \mathrm{~b}$ and is reflected in the $\chi^{2}$ values and is appreciable by zooming into the fitted curves, as shown in figure 2a. Even though the choice of the model seems arbitrary, the off-specular measurements, discussed later, clearly point to a bigger interfacial region than a simple 3-layer model with Gaussian-type roughness would suggest. The recorded intensity requires a much larger scattering volume, than the one predicted by the Gaussian roughness alone.

However, the extended interface width, which has to be added to accurately reproduce the NR is not a slab as it corresponds to roughness comparable to the thickness. This way we cannot gain real quantitative information about the extent of the h-PMMA/d-PS interface. It only becomes possible if one uses the asymmetric tanh function (Ref. [15], p. 22-24, figure 2.13, Eqs. 2.52-2.61). We have fitted this asymmetric tanh function to the four-layer model as shown in figure $3 \mathrm{~b}$ and extracted the width as described by Eq. 2.61 in Ref. [15]. All fitting results are shown in table1. 


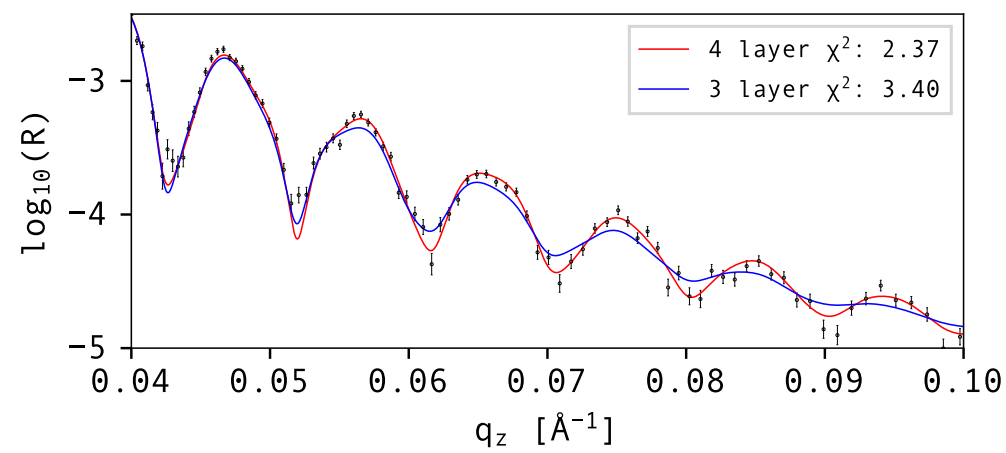

(a)

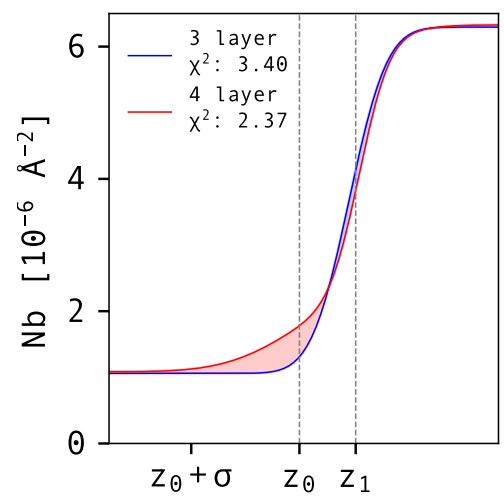

(b)

Figure 2: (a) Zoom of specular reflectivity for sample C using a 3-layer and a 4-layer model, clearly showing the reproduction of features by the latter. (b) Comparison of the interfacial region for sample $\mathrm{C}$ of the two scattering length density profiles, from 3-layer and 4-layer models, that elsewhere look identical. $z_{1}-z_{0}$ is the thickness of the interfacial region in the 4-layer model and $z_{1}-\left(z_{0}+\sigma\right)$ is the total extent of the interface.

In order to estimate this interface width theoretically we have used the following parameters: For our experimental system of $\mathrm{Si} / \mathrm{SiO}_{2}$ / d-PS / h-PMMA / Air, we can estimate the Hamaker constant between two macroscopic phases (1: air) and (3: PS), separated by a medium (2: PMMA), with the Lifshitz theory [21, 9] to be $A_{\text {eff }}=-1.56 \times 10^{-20} \mathrm{~J}$.

The interfacial tension $\gamma$ between PS and PMMA can be estimated using the the self-consistent field theory reported by Helfand [11] as done in ref. [9], and one obtains $\gamma=1.9 \mathrm{~mJ} / \mathrm{m}^{2}$.

The resulting interface width using literature values is plotted as a blue line in figure 3a. As can be seen the polymer/polymer interface width follows the predicted behaviour from capillary wave theory and the measured widths are in line with previous reports on the same system [9, 10] or for PMMA/PS widths for unstable trilayer systems at short annealing times before dewetting [22, 23]. 


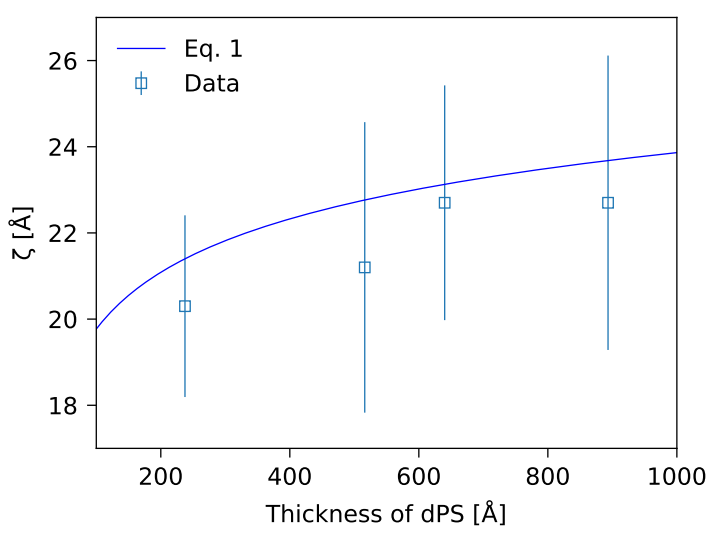

(a)

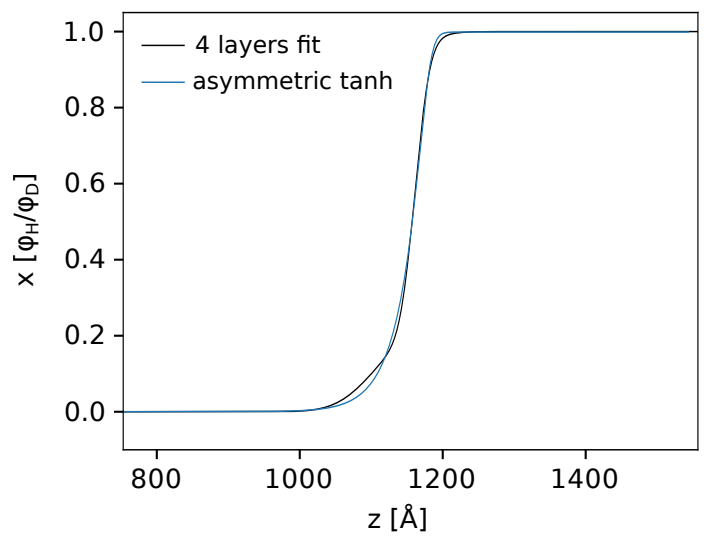

(b)

Figure 3: a) Roughness (points) of the interface between d-PS/h-PMMA as a function of d-PS layer thickness. The solid blue line is calculated using eq.1 using literature values of the $\mathrm{FH}$ interaction parameter, interfacial tension and Hamaker constant to estimate the high q and low q values of the capillary waves cut-offs. The latter cut-off is assumed to originate from vdW interactions. b) h-PMMA vertical monomer density profile for sample $\mathrm{D}$ as a function of distance from the surface (black line) and an example asymmetric tanh fit (blue line).

\subsection{Off-specular reflectometry}

3.2.1. Theory In-plane correlation lengths can be extracted with the use of the distorted-wave Born approximation (DWBA) [20], assuming in-plane exponentially decaying correlations of scattering length density (SLD) deviations at distances greater than the correlation length $\xi$. The consequence of this correlation length in real space is a cut-off at a given wavevector $q_{\min }=2 \pi / \xi[3$.

In brief, the DWBA OSS cross section of a stratified system with $N$ layers:

$$
\left(\frac{d \sigma}{d \Omega}\right)_{\mathrm{OS}}=S_{\mathrm{OS}} \sum_{l l^{\prime}}^{N} G_{l l^{\prime}}^{\perp}\left(p_{f}, p_{i}\right) G_{l l^{\prime}}^{\|}\left(\boldsymbol{q}_{x}\right) .
$$

comprises the weighted double sum of products of the dimensionless perpendicular formfactor, $G_{l l^{\prime}}^{\perp}\left(p_{f}, p_{i}\right)$, accounting for neutron wave distortions due to the optical potential of layers, and the lateral form-factor, $G_{l l^{\prime}}^{\|}\left(\boldsymbol{q}_{x}\right)$. The double sum goes over all combinations of interfaces present in the system $\left(0<l, l^{\prime}<N\right)$. When $l=l^{\prime}$ scattering arises from a) correlated in-plane roughness at the $l^{\text {th }}$ interface that could arise from e.g. capillary waves [24] or b) due to in-plane SLD inhomogeneities across the bulk of layer $l$ due to e.g. holes [25]. The terms with $l \neq l^{\prime}$ are only non-zero if there is out-of-plane correlation among SLD deviations in different layers, which is not significant in the here studied system and will be thus not further discussed. $S_{\mathrm{OS}}$ is the area of the sample illuminated by the neutron beam.

Important to note at this point is that the OSS scattering depends on both the transverse and in-plane form factors. Only the latter is a function of the in-plane momentum transfer, which is dominated by the component along the beam due to the highly 
asymmetric coherence ellipsoid in grazing incidence geometry: $q_{x}=2 \pi / \lambda \times\left(\cos \left(\theta_{\mathrm{f}}\right)-\right.$ $\left.\cos \left(\theta_{\mathrm{i}}\right)\right)$, with the angle of incidence $\theta_{\mathrm{i}}$ and the angle of scattering $\theta_{\mathrm{f}} \neq \theta_{\mathrm{i}}$ and the neutron wavelength $\lambda$. It is basically the Fourier transformation of the pair correlation function $g_{l l^{\prime}}$ of the SLD variations parallel to the interface (averaged over the $y$-direction) and hence very similar to the usual scattering function in the Born approximation (apart from the reduced dimensionality!):

$$
G_{l l^{\prime}}^{\|}\left(q_{x}\right)=d_{l} d_{l^{\prime}} \lambda \int d x e^{-i q_{x} x} g_{l l^{\prime}}(x, 0) .
$$

Throughout this work we have used only an exponentially decaying pair correlation function:

$$
g_{l l^{\prime}}(x, 0)=\left|\Delta \overline{S L D}_{l l^{\prime}}\right|^{2} \exp \left(-|x| / \xi_{l}\right),
$$

with $\triangle \overline{S L D}_{l l^{\prime}}$ being either the SLD difference between two adjacent layers in the case of correlated roughness or the SLD difference of inhomogeneities inside the layer from the average layer SLD. $d_{l}$ and $d_{l^{\prime}}$ correspond to the interface roughness in the case of correlated roughness and to the layer thicknesses in case the SLD deviations perturb the whole layer.

The former orthogonal form factor, $G_{l l^{\prime}}^{\perp}\left(p_{f}, p_{i}\right)$, on the other hand, only depends on the wave vector projections normal to the interface: $p_{i}=2 \pi / \lambda \times \sin \theta_{\mathrm{i}}$ and $p_{f}=2 \pi / \lambda \times \sin \theta_{\mathrm{f}}$. We refer the interested reader to Ref. [20] for a detailed description of this form factor. It is mainly composed of the Fresnel transmission and reflection amplitudes at all interfaces and oscillating Laue functions depending on all layer thicknesses. It thus cannot provide any information about the form of the in-plane structure, apart from information about the scattering contrast of the in-plane inhomogeneities. This does not mean, however, that this form factor cannot produce a multitude of features along the parallel wave vector transfer as the latter is intimately linked with the wave vector projections:

$$
q_{x}=\left[(2 \pi / \lambda)^{2}-p_{f}^{2}\right]^{1 / 2}-\left[(2 \pi / \lambda)^{2}-p_{i}^{2}\right]^{1 / 2} .
$$

Thus, depending on the way of measuring the OSS intensity, all these parameters may be varied and $1 \mathrm{~d}$ or $2 \mathrm{~d}$ plots of OSS intensity can only be projections of the 3 -dimensional space $\left(\lambda, \theta_{\mathrm{i}}, \theta_{\mathrm{f}}\right)$. Therefore analyzing OSS data using $2 \mathrm{~d}$ or even $1 \mathrm{~d}$ projections comes with a high risk of confusion as perpendicular and parallel form factors are getting mixed.

Another important note at this point is that the algorithm used here to analyze OSS data does not include any arbitrary scaling factor, but the reflected intensity can be directly computed (within the DWBA). As can be seen from eqs.2, 3 and 4 the reflected intensity is mainly proportional to the squares of the SLD contrast and the layer thickness or roughness and linearly to the in-plane correlation length. So part of the absolute intensity in OSS comes directly from the in-plane structure, namely the correlation length and the SLD difference in the case of bulk inhomgeneities and therefore part of the structural information is included in the absolute intensity, which would be lost if arbitrary scaling were used. 
3.2.2. OSS experimental results Even though most of the relevant quantitative results for now come from the specular fits, off-specular measurements were crucial to confirm these, as tiny deviations of the SLD could be dismissed during the fitting process of specular data only: when the SLD deviations come from the thickness of the whole layer, they can only marginally change the SLD of a layer in the specular model, but, as discussed above, the contribution to OSS can be quite significant in case of thick layers due to the OSS intensity being proportional to the square of the layer thickness containing the inhomogeneities. One good example of the higher sensitivity of OSS, is the structure of the capping h-PMMA layer in the present system. The specular fits revealed a SLD value very close to the nominal one $\left(1.059 \times 10^{-6} \AA^{-2}\right)$ with a relatively large error around $0.05 \times 10^{-6} \AA^{-2}$ (see table 1 ). However, as will be shown later, the OSS maps exhibit visible off-specular signal originating from this layer. This clearly demonstrates that in-plane inhomgeneities are present in the h-PMMA layer. This is corroborated by atomic force microscopy (AFM) images on these samples revealing hole formation shown in Fig. S1 in the supplementary information (SI).

The top surface shows aligned features in the form of holes. We suspect that these are a consequence of replication of roughness from either the glass slides, which were used for floating the top layer or the silicon wafers. This could potentially be improved by spin coating on mica, which does not need to be be polished. Actually, concerning polar forces the more stable system would be the inverted system with the PMMA in contact with the silicon support due to the higher polar forces of PMMA as explained in our previous work [20]. Therefore in the current system any defect would tend to grow. Concerning specular NR these holes stay invisible as their concentration $c$ is very low $(c<5 \%)$ and thus would not change the mean SLD within the fitting error.

Quantitative fits of the 2D OSS maps (see e.g. figure $5 \mathrm{~b}$ indeed reveal correlation lengths $\left(\xi_{\text {hPMMA/air }}\right)$ on the order of $0.8-2 \mu \mathrm{m}$ (see figure 4 ) of air inclusions inside h-PMMA at a coverage around 5\%. This fits nicely the radius of holes observed in AFM images (Fig. S1 in the SI). The values appear to be more or less constant with a slight tendency of increased size for thicker PS films, which could be an indication of the decreased stability of the top h-PMMA layer when influenced by an increased PS bottom layer [26].

In addition to the onset of dewetting of the top layer an accompanying dewetting type scenario of the bottom layer had to be assumed as well [20] to quantitatively fit the OSS maps. The model assumed here consists of d-PS filled (cylindrical shaped) droplets inside the interfacial layer surrounded by h-PMMA at a concentration derived from the mean SLD of this additional layer between d-PS and h-PMMA. The best OSS fit for e.g. sample D was achieved using a correlation length of $\xi_{\text {interface }}=(1.6 \pm 0.2) \mu \mathrm{m}$, similar to the hole size in the top layer.

The other information only accessible by OSS is the in-plane correlation length at the buried symmetric polymer/polymer interface. As an example, the correlation length for sample D, consisting of a $d_{\mathrm{dPS}}=516 \AA$ thick d-PS layer is $\xi_{\mathrm{dPS} / \mathrm{hPMMA}}=(280 \pm 30) \mathrm{nm}$. The correlation lengths for the other samples will be discussed in a further publication. 

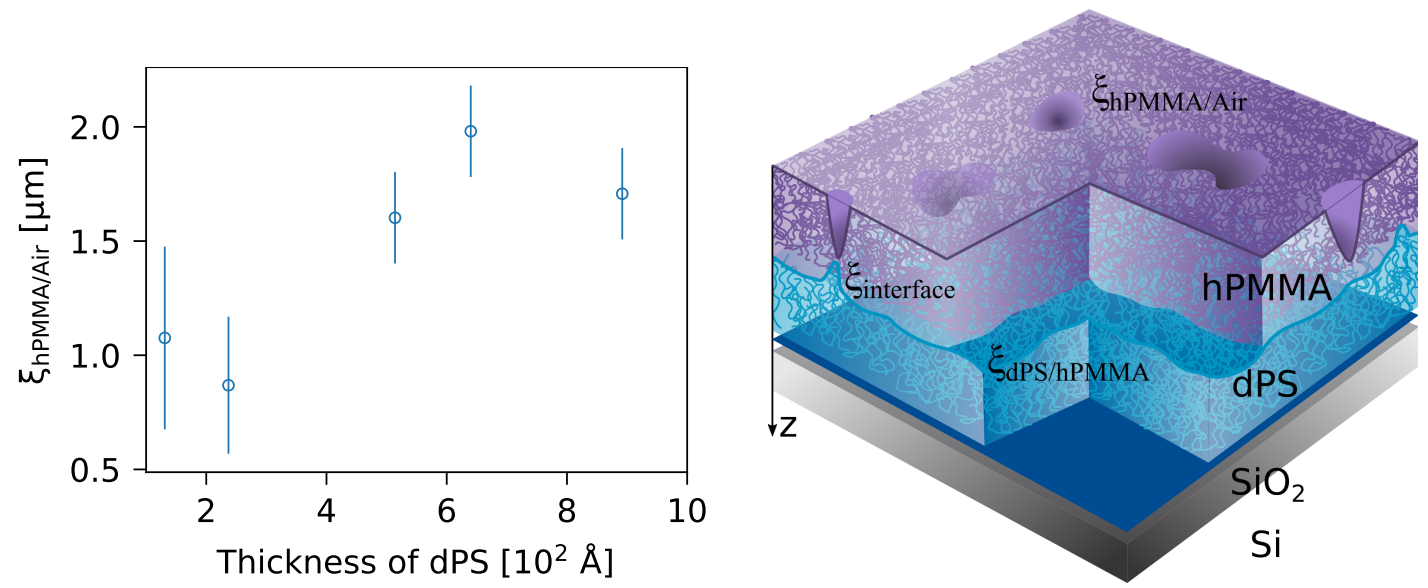

Figure 4: Left: Correlation lengths $\xi_{\text {hPMMA/air }}$ extracted from the OSS fits (circles). This parameter is related to the radius of the holes in the top layer as sketched on the right. Right: Sketch of the model used in this work. $\xi_{\text {interface }}$ is related to the radius of the droplets of d-PS forming at the interface between the dPS and the hPMMA layers, while $\xi_{\mathrm{dPS} / \mathrm{hPMMA}}$ corresponds to the in-plane roughness correlation length of this interface at equilibrium.

Therefore the final OSS model consists of three contributions each with its own correlation length as sketched in Fig. 4 on the right resulting in the total OSS map as shown in Fig. $5 \mathrm{~b}$ for the example of sample D. Each of the three contributions has its particular shape and will be discussed in the next section.

\section{Unraveling off-specular features}

The scattering from the holes discussed in the previous section is mostly concentrated in the high-wavelength region, close to the h-PMMA critical angle, and decreases quickly at higher $q_{z}$. Without this contribution, the shape close to the specular beam at high wavelengths cannot be correctly reproduced, as seen in the two images in the centre and on the right of figure $5 \mathrm{a}$, which lack the aforementioned contribution. The specular ridge is surrounded by a low width component of the diffuse scattering at higher wavelengths. Due to the width of this component being wavelength dependent, it cannot be a geometrical property of the beam and must come from the sample itself. As it turns out, each layer with its respective contrast influences a different part of the OSS spectrum, its position roughly being defined by the corresponding critical wavelength. The position of the features is therefore qualitatively indicative of the source of the perturbation in the sample, giving rise to the intensity in the off-specular spectrum. The total simulated spectrum consists of three contributions to OSS: 1) the scattering from the perturbation caused by holes (filled with air) in the top h-PMMA layer as discussed above, 2) the scattering from droplets of d-PS inside the h-PMMA layer at the polymer/polymer interface due to dewetting of the d-PS and finally 3 ) the interface of interest, between h-PMMA and d-PS.

The first contribution of holes inside h-PMMA scales with $d^{2}$, where $d$ is the thickness 

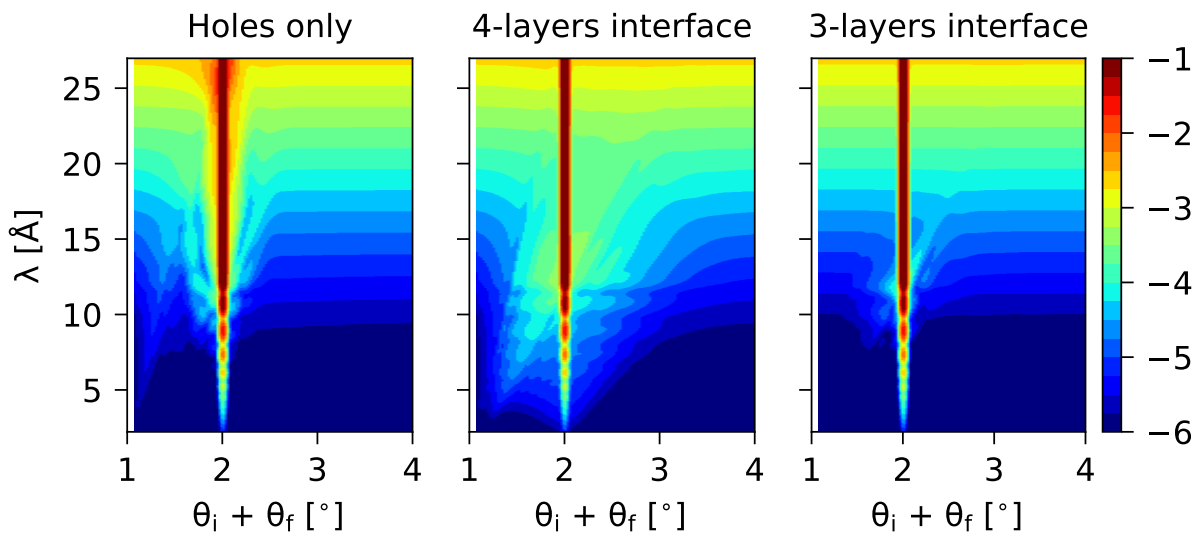

(a)

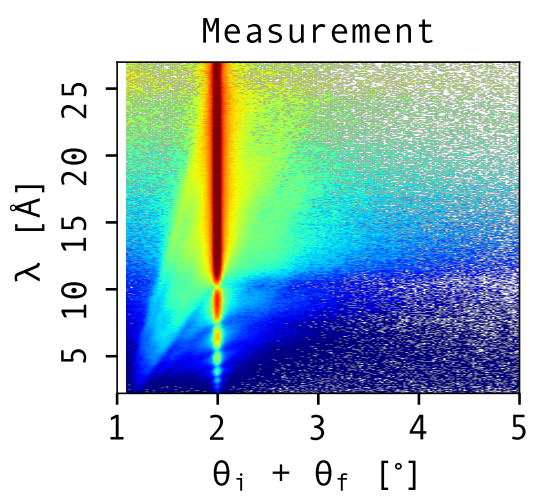

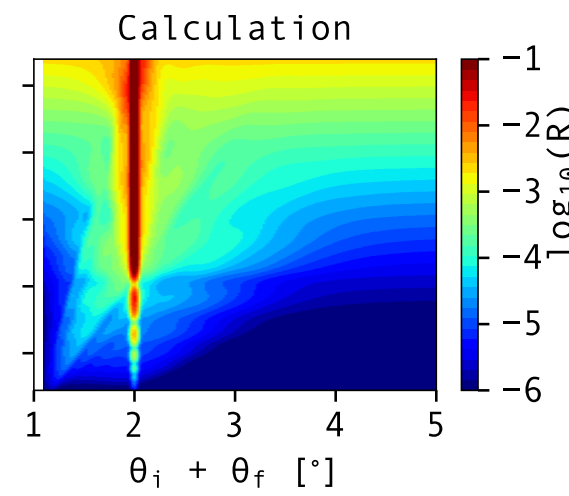

(b)

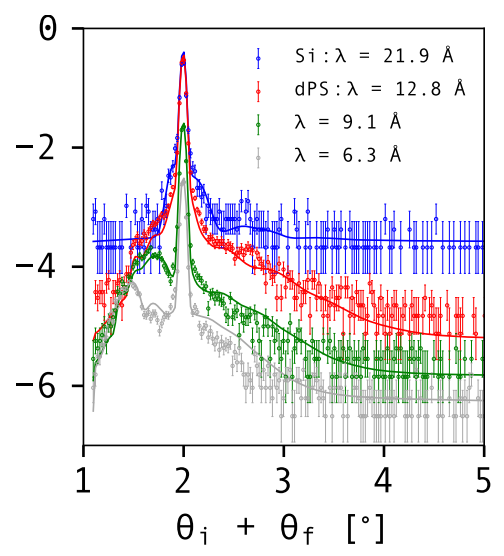

(c)

Figure 5: (a) Calculation of the different contributions to the OSS from the simulation of sample A with a d-PS film of $d_{\mathrm{dPS}}=892 \AA$ : Left) Off-specular intensity coming from the holes in the top hPMMA layer only. Area concentration of holes is $1 \%$. Center) Off-specular intensity is coming from the extended interface between d-PS and h-PMMA only, meaning an increased scattering volume. Right) Off-specular intensity is only coming from the Gaussian roughness $\sigma$ at the interface between d-PS and h-PMMA, as predicted by the 3-layer model. (b) An example of the measured and total simulated scattering from sample D, thickness of d-PS: $d_{\mathrm{dPS}}=516 \AA$. On the left the $2 \mathrm{D}$ measurement is plotted while on the right the calculation as described in the text is shown. (c) Cuts of the experimental 2D map (points) and the fits (lines) of the off-specular scattering from (b) for different wavelengths (see inset for these values).

of h-PMMA. Therefore, already a very small concentration of holes causes huge contribution to the spectrum, as $d \approx 1100 \AA$.

The other source of off-specular scattering is located at the interface of interest between the h-PMMA and d-PS. However, the extent of a Gaussian roughness alone is not enough to describe it for the thicker samples. The shape of the curve and its features in the spectrum are of course correctly described even by the Gaussian roughness from a 3-layer model (figure 5a right), since they come from the same wave field modulation. However, their absolute intensity is almost an order of magnitude weaker, as their intensity scales with $\sigma^{2}$. This is much less than the real total extent of the interface, which was described 
by adding a layer of mixed h-PMMA and d-PS of thickness $d_{\text {mixed }}$ (shown before in figure 2b). By considering a larger scattering volume, the features line up with the experimental data in the low-wavelength region (high $q_{z}$ ), which is dominated by the critical reflection of d-PS, and therefore the information coming from its interface. The contribution to off-specular intensity coming from the extended interface including the polymer/polymer roughness is shown in figure 5 a centre.

Finally, the full, fitted spectrum is shown in figures $5 \mathrm{~b}$ in $\left(\lambda, \theta_{\mathrm{i}}+\theta_{\mathrm{f}}\right)$ space. As shown, it is a combination of the two contributions, the holes in the top layer, correctly describing the high-wavelength region, and the extended interfacial region, correctly describing the low-wavelength region. It has to be noted that, if fitting the OSS in arbitrary scale, one can easily be misled by the nicely aligned features described already by the Gaussian roughness from the 3-layer model.

Here we come back to the important note made in the theoretical OSS section about the intermixed optical and physical features constituting typical OSS patterns, in particular for the analysis of the time-of-flight data. If we take for example the simulation of the OSS from a purely Gaussian interface roughness as simulated in figure $5 \mathrm{a}$ right and we look at a cut along $\left(\theta_{\mathrm{i}}+\theta_{\mathrm{f}}\right)$ at a constant wavelength, e.g. $10 \AA$, we would observe peaks close to the specular ridge stemming from the optical enhancements of the thickness oscillations in the specular signal. If we would simply convert the x-axis in $q_{x}$ of the time-of-flight data and fit the peak positions as $q_{x}^{\max }$ in order to get quantitative in-plane lengths via $\xi=2 \pi / q_{x}^{\max }$ then we would end up with apparent correlation lengths in the range from 5 to $15 \mu \mathrm{m}$ in our case depending on the wavelength chosen, as the strongest optical enhancements are naturally very close to the specular ridge and thus at small $q_{x}$ values. These values are much larger than the hole sizes of around $1 \mu \mathrm{m}$, extracted with the quantitative fits used in this study (see figure 4 ) and also much larger than the roughness correlation lengths at the polymer/polymer interface that are in the sub- $\mu \mathrm{m}$ range.

\section{Conclusion}

We have used bilayers of immiscible polymers, namely h-PMMA on top of d-PS of different thicknesses supported by silicon wafers as model systems for the validation of our newly developed off-specular scattering algorithm. We have managed not only to correctly identify the individual contributions to the scattering pattern, but also to quantitatively disentangle the effects from the total spectrum.

The off-specular scattering yields additional information compared to the wellestablished specular reflectometry technique, which not only improves the specular fits, but also influences their general outcome and physical interpretation. Sometimes, this can mean that a significant departure from the originally assumed simple model is necessary. In our case, slicing the system into a 3-layer model, silicon oxide, d-PS and h-PMMA could easily be considered good enough, if only looking at specular reflectivity. However, by fitting both the specular and off-specular part at the same time, it is 
clear that, there is simply not enough scattering volume (considering only a Gaussian interface) between the two polymers, to correctly describe the results. This study opens up new possibilities for the application of off-specular reflectometry to systems for which little prior information is available, as, by constraining the intensity to the structural parameters (just like in specular reflectometry) and their ratio to the specular peak, it significantly improves the robustness of specular fits.

\section{Acknowledgements}

The authors thank BELSPO for supporting the PhD studentship for AH via the participation of Belgium to the ILL. This work was supported by the Fonds de la Recherche Scientifique - FNRS Grant n. J.0103.17. We are also grateful for the beam time allocation on D17 (https://doi.ill.fr/10.5291/ILL-DATA.9-11-1782). We thank the PSCM for complementary measurements, notably AFM.

\section{References}

[1] Lauter V, Lauter H, Glavic A and Toperverg B 2016 Off-Specular Scattering, and GISANS Neutrons Reference module in materials science and materials engineering (Elsevier, Oxford)

[2] Wang S 1999 Polymers in confined environments (Adv. Polymer Sci. vol 138) (Springer) pp 227275

[3] Seo Y S, Koga T, Sokolov J, Rafailovich M, Tolan M and Sinha S 2005 Physical review letters 94 157802

[4] Jiang Z, Kim H, Jiao X, Lee H, Lee Y J, Byun Y, Song S, Eom D, Li C, Rafailovich M H, Lurio L B and Sinha S K 2007 Physical Review Letters 98

[5] Zhou Y, He Q, Zhang F, Yang F, Narayanan S, Yuan G, Dhinojwala A and Foster M D 2017 ACS Macro Letters 6 915-919 (Preprint https://doi.org/10.1021/acsmacrolett.7b00459) URL https://doi.org/10.1021/acsmacrolett.7b00459

[6] Ocko B, Wu X, Sirota E, Sinha S and Deutsch M 1994 Physical review letters 72242

[7] de Gennes P G 1980 The Journal of Chemical Physics 72 4756-4763 (Preprint https://doi.org/ 10.1063/1.439809) URL https://doi.org/10.1063/1.439809

[8] Rowlinson J S and Widom B 1982 Molecular theory of capillarity (Oxford: Oxford)

[9] Sferrazza M, Xiao C, Jones R A L, Bucknall D G, Webster J and Penfold J 1997 Phys. Rev. Lett. 78(19) 3693-3696 URL https://link.aps.org/doi/10.1103/PhysRevLett.78.3693

[10] Seeck O, Kaendler I, Tolan M, Shin K, Rafailovich M, Sokolov J and Kolb R 2000 Applied Physics Letters 76 2713-2715

[11] Helfand E and Tagami Y 1972 The Journal of Chemical Physics 56 3592-3601

[12] Jablin M S, Zhernenkov M, Toperverg B P, Dubey M, Smith H L, Vidyasagar A, Toomey R, Hurd A J and Majewski J 2011 Physical Review Letters 106(13) 138101 URL http: //link.aps.org/doi/10.1103/PhysRevLett.106.138101

[13] Liesche G 2012 Flow behaviour of polymer melts and solutions at the solid-liquid interface Bachelor's thesis Hochschule Bremerhaven

[14] Wilkes C E, Summers J W, Daniels C A and Berard M T (eds) 2005 PVC Handbook (Munich ; Cincinnati: Hanser) ISBN 978-3-446-22714-9 978-1-56990-379-7

[15] Tolan M 1998 X-Ray Scattering from Soft-Matter Thin Films, -Materials Science \& Basic Research -, STMP (Springer)

[16] Saerbeck T, Cubitt R, Wildes A, Manzin G, Andersen K H and Gutfreund P 2018 Journal of Applied Crystallography 51 249-256 URL https://doi.org/10.1107/S160057671800239X 
[17] Gutfreund P, Saerbeck T, Gonzalez M A, Pellegrini E, Laver M, Dewhurst C and Cubitt R 2018 Journal of Applied Crystallography 51 606-615 URL https://doi.org/10.1107/ S160057671800448X

[18] Richard D, Ferrand M and Kearley G 1996 Journal of Neutron Research 4 33-39

[19] Adlmann F A, Pálsson G K, Bilheux J, Ankner J, Gutfreund P, Kawecki M and Wolff M 2016 Journal of Applied Crystallography 49 2091-2099

[20] Hafner A, Gutfreund P, Toperverg B P, Jones A O F, de Silva J P, Wildes A, Fischer H E, Geoghegan M and Sferrazza M 2021 Journal of Applied Crystallography 54 924-948 URL https://doi.org/10.1107/S1600576721003575

[21] Israelachvili J N 2011 Intermolecular and Surface Forces third edition ed (Amsterdam: Elsevier, Academic Press) ISBN 978-0-12-391927-4 978-0-12-375182-9 oCLC: 249272556

[22] de Silva J P, Martin S J, Cubitt R and Geoghegan M 2009 EPL 8636005 URL http://stacks. iop.org/0295-5075/86/i=3/a=36005

[23] de Silva J P, Cousin F, Wildes A R, Geoghegan M and Sferrazza M 2012 Phys. Rev. E 86(3) 032801 URL https://link.aps.org/doi/10.1103/PhysRevE.86.032801

[24] James D, Higgins A M, Rees P, Geoghegan M, Brown M R, Chang S S, Môn D, Cubitt R, Dalgliesh $\mathrm{R}$ and Gutfreund P 2015 Soft matter 11 9393-9403

[25] Castel A, Gutfreund P, Cabane B and Rharbi Y 2020 Soft matter 16 1485-1497

[26] de Silva J P, Geoghegan M, Higgins A M, Krausch G, David M O and Reiter G 2007 Phys. Rev. Lett. 98(26) 267802 URL https://link.aps.org/doi/10.1103/PhysRevLett.98.267802 